\title{
Locally Induced Predictive Models
}

\author{
Ulf Johansson, Tuve Löfström, Cecilia Sönströd \\ School of Business and Informatics \\ University of Borås \\ Borås, Sweden \\ \{ulf.johansson, tuve.lofstrom, cecilia.sonstrod\}@hb.se
}

\begin{abstract}
Most predictive modeling techniques utilize all available data to build global models. This is despite the wellknown fact that for many problems, the targeted relationship varies greatly over the input space, thus suggesting that localized models may improve predictive performance. In this paper, we suggest and evaluate a technique inducing one predictive model for each test instance, using only neighboring instances. In the experimentation, several different variations of the suggested algorithm producing localized decision trees and neural network models are evaluated on $30 \mathrm{UCI}$ data sets. The main result is that the suggested approach generally yields better predictive performance than global models built using all available training data. As a matter of fact, all techniques producing $\mathrm{J48}$ trees obtained significantly higher accuracy and AUC, compared to the global J48 model. For RBF network models, with their inherent ability to use localized information, the suggested approach was only successful with regard to accuracy, while global RBF models had a better ranking ability, as seen by their generally higher AUCs.

Index Terms-Local learning, Predictive modeling, Decision
\end{abstract} trees, RBF networks

\section{INTRODUCTION}

Exploiting available data in order to extract useful and actionable information is the overall goal of the generic activity termed data mining. Some data mining techniques directly obtain the information by performing a descriptive partitioning of the data. More often, however, data mining techniques utilize stored data in order to build predictive models.

Predictive modeling combines techniques from several disciplines. Many algorithms and techniques come from machine learning, i.e., the sub-field of artificial intelligence focused on programs capable of learning. In the data mining context, learning most often consists of obtaining a (general) model from examples, i.e., data instances where the value of the target variable is known. Since the purpose of all predictive modeling is to apply the model on novel data (a test or production set), it is absolutely vital that the model generalizes well to unseen data. More precisely, for predictive classification, the number of incorrect classifications (the error rate) should be minimized - or the number of correct classifications (the accuracy) should be maximized.

As described above, predictive modeling normally consists of a two-step process: first an inductive step, where the model is constructed from the data, and then a second, deductive, step where the model is applied to the test instances. An alternative approach is, however, to omit the model building entirely and directly classify novel instances based on available training instances. Such approaches are called lazy learners or instance based learners. The most common lazy approach is nearest neighbor classification, which classifies a test instance based on the majority class among the $k$ closest (according to some distance measure) training instances. The value $k$ is a parameter to the algorithm, and the entire technique is known as $k$-Nearest Neighbor (kNN). kNN, consequently, does not, in contrast to techniques like neural networks and decision trees, use a global model covering the entire input space. Instead, classification is based on local information only.

Model building is clearly data-driven, making the validity of the process dependent on some basic, although most often not explicitly expressed, assumptions. Most importantly, the instances used for the actual training of the model must be good examples to learn from. Despite this, most techniques simply use all available instances for model training, thus prioritizing the global aspects of the model, while possibly ignoring important local relationships. In this paper, we apply the localized aspect of instance based learning to predictive modeling. More specifically, we evaluate different algorithms building localized decision tree and neural network models by using only a subset of the available training data. The main idea suggested here is to use neighboring instances to build one model for each test instance.

\section{BACKGROUND}

A predictive classification model is a function $f$ mapping each instance $\boldsymbol{x}$ to one label in a predefined set of discrete classes $\left\{c_{1}, c_{2}, \ldots, c_{n}\right\}$. Normally, predictive models are obtained by applying some supervised learning technique on historical (training) data. Most supervised learning techniques require that values for the target variable are available for all training instances, i.e., the training instances must be labeled. A training instance, thus, consists of an input vector $\mathbf{x}(i)$ with a corresponding target value $y(i)$. The predictive model is an estimation of the function $y=f(\mathbf{x} ; \theta)$ able to predict a value $y$, given an input vector of measured values $\mathbf{x}$ and a set of estimated parameters $\theta$ for the model $f$. The process of finding the best $\theta$ values is the core of the learning technique.

The use of neighboring instances for the actual classification makes it, in theory, possible for $\mathrm{kNN}$ to produce arbitrarily shaped decision boundaries, while decision trees and rule-based learners are constrained to rectilinear decision boundaries. Standard kNN is a straightforward classification technique that normally performs quite well, in spite of its 
simplicity. Often, standard $\mathrm{kNN}$ is used as a first choice just to obtain a lower bound estimation of the accuracy that should be achieved by more powerful methods see e.g., [1]. Unfortunately, standard $\mathrm{kNN}$ is extremely dependent on the parameter value $k$. If $k$ is too small, the algorithm becomes very susceptible to noise. If $k$ is too large, the locality aspect becomes less important, typically leading to test instances being misclassified based on training instances quite different from the test instance.

\section{A. Related work}

Applying lazy techniques to prediction has been studied both for regression, such as in Quantitative Structure-Activity Relationship (QSAR) modeling in the medicinal chemistry domain [2], [3], and for classification [4], [5], [6], [7]. In the QSAR modeling, the motivation for the local approach is the assumption that similar compounds have similar biological activities, as studied in [8]. One of the first local approaches in QSAR modeling was suggested in [3], where a locally weighted linear regression was utilized in the analysis. In [2], the local regression approach is evaluated and contrasted to the global approach using random forests, support vector machines, and partial least squares models on both simulated and real world data. The results show that the local approach has a relatively high computational cost, high risk in defining locality and no reliable increase in predictive performance, indicating that the local approach might not be very successful for regression in general and for QSAR modeling in particular.

For classification, a number of local approaches have been proposed. Friedman proposed the lazy decision tree algorithm, which builds a decision path for each test instance instead of a global decision tree [9]. The lazy decision tree algorithm has also been utilized in boosted ensembles [4]. A similar approach was employed to build bagged ensembles in [5], with the difference that the trees used were lazy option trees [10]. An option tree is a decision tree in which a given internal node in the tree is split in multiple ways (using multiple attributevalue tests, or "options"). The final probability estimate is the average of the estimates produced by each of these options. In [7], a local approach is used to build a global decision tree where lazy trees for each test instance are built in each leaf. The aim of this global lazy tree is to achieve good ranking capability. All these approaches build a model for each test instance using the complete training set.

A bagging approach for very weak classifiers, which utilizes a local approach similar to the one presented in this paper, can be found in [6]. The algorithm trains an ensemble of bagged very weak models, such as decision stumps, based on locally selected instances. The differences to our approach lies both in the kind of model being built and in how the selection of the nearest neighbors is performed.

\section{METHOD}

As described in the introduction, the overall purpose is to evaluate the effect of using neighboring instances to train one model for each test instance. In particular, we compare the predictive performance of this approach to the standard approach, i.e., using all available data to build one, global, model. For simplicity, and to allow easy replication of the experiments, the Weka workbench [11] is used for the experimentation. For the actual modeling, we elected to use one decision tree technique and one neural network technique. More specifically, the Weka implementation of $\mathrm{C} 4.5$ [12] called $J 48$, and the radial basis function network (RBF) as implemented in Weka were used. During experimentation, all Weka parameter settings were left at the default values.

It must be noted that $\mathrm{J} 48$ trees and RBF networks have some fundamentally different properties which are of importance for this study. More specifically, the J48 technique does not, in any way, explicitly utilize any concepts related to proximity in the attribute space. An RBF network, on the other hand, when trained for classification, first uses the k-means clustering algorithm to provide the basis functions and then learns a logistic regression on top of that. So, simply put, even a standard RBF network trained using all available data is trained to utilize at least some of the locality aspect of the data set. With this in mind, it is quite interesting to compare the results for the two techniques, when used for producing locally trained models.

This study contains two experiments. In the first experiment, the suggested approach is tested by using different percentages of the available training data for model building. Altogether five different fractions are evaluated; $1 \%, 10 \%, 25 \%, 50 \%$, and $100 \%$. So, as an example, when $10 \%$ is used for training J48 models, this means that for each test instance, one J48 tree is built using the $10 \%$ closest instances, to classify just that instance. For all distance calculations, the standard method used for lazy learners in Weka is applied.

In the second experiment, we test two algorithms aimed at automatically selecting, for each test instance, a suitable fraction of the training instances for the model building. This is, of course, similar to finding an appropriate value for the parameter $k$ in $\mathrm{kNN}$. The first algorithm uses standard internal cross-validation to select the percentage of instances to use. For this, the Weka meta technique CVParameter Selection, utilizing ten internal folds was used. For simplicity, the fractions available to choose from were the same as in Experiment, i.e., $1 \%, 10 \%, 25 \%, 50 \%$, and $100 \%$. It must be noted that this selection is performed once per fold, not once per instance.

The second algorithm employs the machine learning technique used to build the model (i.e. $J 48$ or $R B F$ ) to find the appropriate $k$-value using both the evaluated test instance and the nearest neighbors used for training. A model is built using the $k$ nearest neighbors from the training set for each $k$-value between the number of classes +1 up to an upper limit. The upper limits tested were $10 \%$ and $25 \%$ of all instances available. To select which of the local models to use, a probability distribution over the classes is achieved by evaluating the model on the nearest neighbors and the test instance. The model achieving the most certain prediction on any class is selected as the model to use. The probabilities are 
adjusted using Laplace to account for the greater uncertainty when estimating the probabilities on smaller samples, i.e. when $k$ is smaller. For simplicity, these two versions of the algorithm are referred to as A10 and A25.

For the evaluation, predictive performance is measured using both accuracy and area-under-the-ROC-curve (AUC). While accuracy is based only on the classifications, AUC measures the ability to rank instances according to how likely they are to belong to a certain class; see e.g., [13]. AUC can be interpreted as the probability of ranking a true positive instance ahead of a false positive; see [14]. Since 10x10-fold crossvalidation was used, all reported performance measures are averaged over the 100 folds. The 30 data sets used are all publicly available from the UCI Repository [15].

\section{RESUlTS}

Table I below shows accuracy results for J48. One first, important, observation is that the results vary greatly between the data sets. A closer inspection shows that no specific training size is always superior to any other training size. As a matter of fact, all training fractions evaluated obtain the highest accuracy on at least two data sets. In addition, on most data sets, there is no clear trend; i.e., the pattern is neither the less data used the higher accuracy nor the more data used the higher accuracy.

TABLE I

EXP. 1: LOCALLY TRAINED J48 MODELS. ACCURACY FOR DIFFERENT TRAINING SIZES

\begin{tabular}{lccccc} 
Data set & $1 \%$ & $10 \%$ & $25 \%$ & $50 \%$ & $100 \%$ \\
\hline bal. scale & .862 & .695 & .795 & .793 & .778 \\
breast cancer & .740 & .717 & .677 & .681 & .743 \\
breast cancer-w & .969 & .961 & .967 & .967 & .950 \\
car & .898 & .924 & .935 & .933 & .922 \\
cmc & .485 & .509 & .503 & .501 & .514 \\
horse-colic & .810 & .837 & .826 & .836 & .852 \\
credit-a & .856 & .848 & .847 & .844 & .856 \\
credit-g & .709 & .713 & .735 & .733 & .712 \\
cylinder-bands & .724 & .694 & .682 & .704 & .578 \\
dermatology & .964 & .963 & .964 & .963 & .941 \\
diabetes-pima & .716 & .728 & .753 & .746 & .745 \\
ecoli & .844 & .824 & .845 & .838 & .828 \\
glass & .700 & .714 & .746 & .757 & .676 \\
haberman & .735 & .730 & .664 & .664 & .722 \\
heart-c & .772 & .801 & .801 & .791 & .771 \\
heart-h & .804 & .787 & .804 & .789 & .802 \\
heart-s & .777 & .787 & .791 & .784 & .782 \\
hepatitis & .814 & .807 & .822 & .821 & .792 \\
ionosphere & .860 & .858 & .913 & .925 & .897 \\
iris & .954 & .951 & .935 & .949 & .947 \\
labor & .843 & .894 & .913 & .884 & .786 \\
liver-disorders & .626 & .622 & .646 & .662 & .658 \\
lymphography & .817 & .831 & .797 & .815 & .758 \\
sonar & .862 & .772 & .800 & .803 & .736 \\
tae & .629 & .536 & .517 & .545 & .574 \\
tic-tac-toe & .976 & .957 & .953 & .931 & .853 \\
vehicle & .706 & .731 & .747 & .751 & .723 \\
vote & .942 & .966 & .963 & .962 & .966 \\
wine & .951 & .961 & .979 & .971 & .932 \\
zoo & .961 & .943 & .935 & .919 & .926 \\
\hline mean & $\mathbf{. 1 0}$ & $\mathbf{. 8 0 2}$ & $\mathbf{. 8 0 8}$ & $\mathbf{. 8 0 9}$ &. $\mathbf{. 9 1}$ \\
mean rank & $\mathbf{2 . 9 0}$ & $\mathbf{3 . 1 0}$ & $\mathbf{2 . 5 0}$ & $\mathbf{2 . 8 3}$ & $\mathbf{3 . 6 7}$ \\
\#wins & $\mathbf{1 0}$ & $\mathbf{2}$ & $\mathbf{1 1}$ & $\mathbf{4}$ & $\mathbf{3}$ \\
& & & & &
\end{tabular}

On the other hand, when looking at overall mean accuracies and ranks, it is obvious that the standard approach (using all available data to build one global model) is clearly the worst. Comparing the different training sizes, using the smallest training set $(1 \%)$, i.e., for some data sets only a handful of instances, is remarkably often the best choice. Somewhat surprisingly, training using only $1 \%$ actually obtained the highest mean accuracy overall. Comparing mean ranks, however, both $25 \%$ and $50 \%$, obtained slightly better results than $1 \%$. To determine statistically significant differences, we compare mean ranks and use the statistical tests recommended by Demšar [16] for comparing several classifiers over a number of data sets, i.e., a Friedman test [17], followed by a BonferroniDunn post-hoc test [18]. With five classifiers, 30 data sets and treating the standard approach (training one global model using all data) as the control, the critical distance (for $\alpha=0.05$ ) is 0.91 . So based on these tests, training one model per test instance using the $25 \%$ closest instances produced significantly more accurate models than the standard approach.

Table II below shows accuracy results for the RBF networks.

TABLE II

EXP. 1: LOCALLY TRAINED RBF MODELS. ACCURACY FOR DIFFERENT TRAINING SIZES

\begin{tabular}{|c|c|c|c|c|c|}
\hline Data set & $1 \%$ & $10 \%$ & $25 \%$ & $50 \%$ & $100 \%$ \\
\hline bal. scale & .756 & .887 & .863 & .879 & .863 \\
\hline breast cancer & .728 & .710 & .715 & .711 & .714 \\
\hline breast cancer-w & .968 & .967 & .960 & .969 & .962 \\
\hline car & .852 & .741 & .894 & .766 & .888 \\
\hline $\mathrm{cmc}$ & .475 & .538 & .495 & .528 & .502 \\
\hline horse-colic & .793 & .791 & .801 & .796 & .803 \\
\hline credit-a & .850 & .821 & .795 & .804 & .796 \\
\hline credit-g & .713 & .742 & .737 & .744 & .737 \\
\hline cylinder-bands & .707 & .766 & .722 & .767 & .727 \\
\hline dermatology & .953 & .961 & .968 & .964 & .966 \\
\hline diabetes-pima & .723 & .740 & .743 & .747 & .740 \\
\hline ecoli & .827 & .818 & .838 & .838 & .833 \\
\hline glass & .700 & .729 & .663 & .710 & 649 \\
\hline haberman & .736 & .716 & .735 & .707 & .731 \\
\hline heart-c & .772 & .815 & .831 & .819 & .835 \\
\hline heart-h & .804 & .814 & .831 & .830 & .828 \\
\hline heart-s & .777 & .787 & .833 & .817 & .831 \\
\hline hepatitis & .814 & .826 & .854 & .861 & .853 \\
\hline ionosphere & .855 & .852 & .915 & .909 & .917 \\
\hline iris & .954 & .953 & .961 & .954 & .960 \\
\hline labor & .843 & .928 & .913 & .912 & .911 \\
\hline liver-disorders & .628 & .641 & .655 & .628 & .651 \\
\hline lymphography & .818 & .845 & .800 & .805 & .797 \\
\hline sonar & .862 & .813 & .760 & .844 & .726 \\
\hline tae & .629 & .607 & .504 & .559 & .498 \\
\hline tic-tac-toe & .984 & .791 & .712 & .787 & .709 \\
\hline vehicle & .709 & .716 & .653 & .720 & .654 \\
\hline vote & .940 & .956 & .931 & .955 & .937 \\
\hline wine & .951 & .984 & .985 & .986 & .977 \\
\hline zOO & .961 & .960 & .947 & .968 & .943 \\
\hline mean & .803 & .807 & .800 & .809 & .798 \\
\hline mean rank & 3.45 & 3.03 & 2.83 & 2.45 & 3.23 \\
\hline \#wins & 6 & 6 & 7 & 8 & 3 \\
\hline
\end{tabular}

Here, too, it is obvious that the standard approach most often results in weaker models than the locally trained models. Looking at the mean accuracy over all data sets, all options using locally trained models outperform the standard approach. All in all, however, considering the mean ranks and using the 
statistical tests described above, the standard approach is not significantly worse than any of the locally trained versions.

Comparing these results to the J48 results, the main difference is that here it seems better to use more data; i.e., the best results overall are obtained using $50 \%$, while using only $1 \%$ is the worst option. Still, the results vary considerably over the different data sets, even if the mean accuracies are quite similar. Specifically, the number of wins are very evenly distributed between the different training fractions.

The AUC results for $\mathrm{J} 48$ models are presented in Table III below. Regarding AUC, the picture is actually quite clear. Somewhat unexpectedly, all locally trained models clearly outperform the standard approach with regard to ranking ability, and it is most often better to use more data $(25 \%$ or $50 \%)$, compared to using fewer training instances $(1 \%$ or $10 \%$ ). Looking at data set wins, using either $25 \%$ or $50 \%$ is the best option on a huge majority of all data sets. The statistical tests also indicate that all training sizes except $10 \%$ obtained significantly higher AUC, compared to the standard approach. In addition, if the Friedman test is instead used to identify all statistically significant dfferences, a Nemenyi post-hoc test [19] indicates that the AUC obtained by the locally trained models using either $25 \%$ or $50 \%$ was significantly higher than for all three other J48 setups.

TABLE III

EXP. 1: LOCALLY TRAINED J48 MODELS. AUC FOR DIFFERENT TRAINING SIZES

\begin{tabular}{lccccc} 
Data set & $1 \%$ & $10 \%$ & $25 \%$ & $50 \%$ & $100 \%$ \\
\hline bal. scale & .966 & .813 & .959 & .957 & .845 \\
breast cancer & .657 & .575 & .635 & .648 & .606 \\
breast cancer-w & .978 & .972 & .988 & .989 & .957 \\
car & .985 & .983 & .994 & .994 & .981 \\
cmc & .657 & .688 & .702 & .702 & .691 \\
horse-colic & .836 & .842 & .875 & .880 & .843 \\
credit-a & .882 & .894 & .906 & .905 & .886 \\
credit-g & .655 & .653 & .727 & .730 & .647 \\
cylinder-bands & .735 & .759 & .798 & .843 & .500 \\
dermatology & 1.000 & .994 & 1.000 & 1.000 & .982 \\
diabetes-pima & .718 & .742 & .797 & .793 & .751 \\
ecoli & .980 & .963 & .981 & .979 & .963 \\
glass & .798 & .823 & .915 & .916 & .794 \\
haberman & .600 & .647 & .618 & .613 & .568 \\
heart-c & .832 & .830 & .877 & .868 & .769 \\
heart-h & .840 & .847 & .864 & .858 & .775 \\
heart-s & .835 & .802 & .866 & .858 & .786 \\
hepatitis & .678 & .752 & .845 & .841 & .668 \\
ionosphere & .895 & .851 & .958 & .971 & .891 \\
iris & 1.000 & 1.000 & .993 & 1.000 & .990 \\
labor & .844 & .906 & .976 & .953 & .726 \\
liver-disorders & .645 & .597 & .680 & .713 & .650 \\
lymphography & .839 & .838 & .789 & .789 & .785 \\
sonar & .859 & .789 & .895 & .900 & .753 \\
tae & .745 & .760 & .693 & .738 & .747 \\
tic-tac-toe & .996 & .984 & .990 & .980 & .901 \\
vehicle & .719 & .761 & .844 & .852 & .762 \\
vote & .966 & .984 & .990 & .988 & .979 \\
wine & .979 & .977 & 1.000 & .999 & .968 \\
zoo & 1.000 & 1.000 & 1.000 & 1.000 & 1.000 \\
\hline mean & .837 & $\mathbf{. 8 3 4}$ & $\mathbf{. 8 7 2}$ & $\mathbf{. 8 7 5}$ & $\mathbf{. 8 0 5}$ \\
mean rank & $\mathbf{3 . 2 7}$ & $\mathbf{3 . 5 2}$ & $\mathbf{1 . 9 2}$ & $\mathbf{1 . 9 8}$ & $\mathbf{4 . 3 2}$ \\
\#wins & $\mathbf{6}$ & $\mathbf{3}$ & $\mathbf{1 4}$ & $\mathbf{1 3}$ & $\mathbf{1}$ \\
& & & & & \\
& & & & &
\end{tabular}

Looking at the AUC results for RBF models in Table IV below, the overall situation is very different. For RBF models, comparing mean ranks, the best ranking ability is actually produced by using all available data when training the model. Based on statistical testing, using all data produced RBF networks with significantly higher AUC than RBF models trained using either $1 \%$ or $10 \%$.

TABLE IV

EXP. 1: LOCALLY TRAINED RBF MODELS. AUC FOR DIFFERENT TRAINING SIZES

\begin{tabular}{|c|c|c|c|c|c|}
\hline Data set & $1 \%$ & $10 \%$ & $25 \%$ & $50 \%$ & $100 \%$ \\
\hline bal. scale & .905 & .960 & .971 & .982 & .971 \\
\hline breast cancer & .624 & .635 & .692 & .663 & .687 \\
\hline breast cancer-w & .973 & .976 & .988 & .985 & .990 \\
\hline car & .962 & .988 & .982 & .984 & .979 \\
\hline $\mathrm{cmc}$ & 633 & .732 & .680 & .726 & .681 \\
\hline horse-colic & .812 & .782 & .830 & .832 & .835 \\
\hline credit-a & .869 & .877 & .855 & .897 & .875 \\
\hline credit-g & .618 & .751 & .760 & .762 & .756 \\
\hline cylinder-bands & .764 & .768 & .789 & .822 & .808 \\
\hline dermatology & .998 & .998 & 1.000 & .997 & .999 \\
\hline diabetes-pima & .711 & .800 & .791 & .809 & .789 \\
\hline ecoli & .971 & .954 & .976 & .966 & .976 \\
\hline glass & .797 & .838 & .830 & .868 & .827 \\
\hline haberman & 618 & .606 & .661 & 614 & .652 \\
\hline heart-c & .832 & .840 & .898 & .886 & .900 \\
\hline heart-h & .840 & .820 & .878 & .881 & .887 \\
\hline heart-s & .837 & .804 & .891 & .880 & .895 \\
\hline hepatitis & .678 & .627 & .851 & .758 & .878 \\
\hline ionosphere & .895 & .853 & .940 & .911 & .954 \\
\hline iris & 1.000 & 1.000 & 1.000 & 1.000 & 1.000 \\
\hline labor & .844 & .971 & .930 & .931 & .951 \\
\hline liver-disorders & .646 & .646 & 679 & .652 & .680 \\
\hline lymphography & .824 & .858 & .824 & .857 & .868 \\
\hline sonar & .859 & .826 & .811 & .847 & .812 \\
\hline tae & .713 & .756 & .680 & .735 & .686 \\
\hline tic-tac-toe & .994 & .925 & .731 & .928 & .739 \\
\hline vehicle & .661 & .798 & .786 & .829 & .789 \\
\hline vote & .968 & .963 & .970 & .975 & .978 \\
\hline wine & .979 & .996 & .989 & .985 & .993 \\
\hline zoo & 1.000 & 1.000 & .994 & 1.000 & .995 \\
\hline mean & .828 & .845 & .855 & .865 & .861 \\
\hline mean rank & 4.05 & 3.27 & 2.98 & 2.40 & 2.30 \\
\hline \#wins & 4 & 7 & 5 & 10 & 12 \\
\hline
\end{tabular}

Summarizing the first experiment, the overall picture is that the suggested approach of training one model per instance using only local instances is almost always a better choice than building one global model using all available data. Especially when building J48 models, all smaller training sizes and local models are clearly better than the global model, both with regard to accuracy and AUC. As a matter of fact, specifically using $25 \%$ of the instances for the local training resulted in J48 models that had both significantly higher accuracy and AUC, compared to the global model. For RBF networks, the results are a bit more mixed, but using $50 \%$ to train a local model obtained clearly higher accuracy, and simlar AUC. Table $\mathrm{V}$ below shows the number of wins for the locally trained models, using different training sizes. The tabulated numbers are wins for locally trained models against the global model. In a pair-wise comparison over 30 data sets, 20 wins are sufficient for statistical significance, using a standard sign test. Bold numbers indicate a significant number of wins for the 
local technique while underlined numbers indicate a significant number of wins for the global technique.

TABLE V

EXP. 1 - SUMMARY

\begin{tabular}{lcccc|cccc} 
& \multicolumn{4}{c|}{$\mathrm{J} 48$} & \multicolumn{4}{c}{$\mathrm{RBF}$} \\
& $1 \%$ & $10 \%$ & $25 \%$ & $50 \%$ & $1 \%$ & $10 \%$ & $25 \%$ & $50 \%$ \\
\hline Accuracy & 19 & 19 & $\mathbf{2 1}$ & $\mathbf{2 1}$ & 12 & 16 & 19 & $\mathbf{2 0}$ \\
AUC & $\mathbf{2 1 . 5}$ & $\mathbf{2 1}$ & $\mathbf{2 8 . 5}$ & $\mathbf{2 8 . 5}$ & $\underline{5}$ & 13 & $\underline{10}$ & 14
\end{tabular}

So, also when comparing the techniques head-to-head, all local techniques producing J48 trees obtained significantly higher AUC, compared to the global J48 model. In addition all four training sizes resulted in $19-21$ wins with regard to accuracy; i.e., the difference in accuracy is either statistically significant, or quite clear. Looking at RBF models, the locally trained models using either $25 \%$ or $50 \%$ of the instances obtained 19 and 20 accuracy wins against the global model, respecitively. Comparing AUCs, however, the global technique outperformed all locally trained variants. Against the techniques using either $1 \%$ or $25 \%$, the number of wins was even enough for statistical significance.

Turning to experiment 2, Table VI below shows the accuracy results.

TABLE VI

EXP. 2 - ACCURACY

\begin{tabular}{|c|c|c|c|c|c|c|c|c|}
\hline \multirow[b]{2}{*}{ Data set } & \multicolumn{4}{|c|}{ J48 } & \multicolumn{4}{|c|}{ RBF } \\
\hline & $100 \%$ & A 10 & A 25 & $\mathrm{CV}$ & $100 \%$ & A10 & A25 & $\mathrm{CV}$ \\
\hline bal. scale & .778 & .794 & .797 & .862 & .863 & .880 & .883 & .885 \\
\hline breast cancer & .743 & .748 & .747 & .730 & .714 & .745 & .748 & .708 \\
\hline breast cancer-w & .950 & .969 & .969 & .967 & .962 & .968 & .968 & .968 \\
\hline ar & .922 & .912 & .926 & .921 & .888 & .853 & .814 & .887 \\
\hline $\mathrm{mc}$ & .514 & .490 & .490 & .511 & .502 & .491 & .492 & .531 \\
\hline orse-colic & .852 & .835 & .835 & .848 & .803 & .828 & .827 & .790 \\
\hline & .856 & .873 & .873 & .853 & .796 & .868 & .868 & .849 \\
\hline$g$ & .712 & .741 & .742 & .711 & .737 & .746 & .746 & .742 \\
\hline r-bands & .578 & .704 & .699 & .717 & .727 & .714 & .717 & .761 \\
\hline & .941 & .964 & .968 & .978 & .966 & .968 & .967 & .965 \\
\hline & .745 & .747 & .745 & .736 & .740 & .754 & .750 & .743 \\
\hline coli & .828 & .833 & .843 & .836 & .833 & .858 & .851 & .834 \\
\hline lass & .676 & .698 & .703 & .705 & .649 & .704 & .713 & .71 \\
\hline & .722 & .720 & .737 & .719 & .731 & .705 & .731 & .714 \\
\hline & .771 & .824 & .832 & .793 & .835 & .826 & .833 & .827 \\
\hline & .802 & .812 & .818 & .791 & .828 & .827 & .836 & .819 \\
\hline & .782 & .809 & .811 & .775 & .831 & .805 & .804 & .828 \\
\hline & .792 & .831 & .831 & .796 & .853 & .833 & .838 & .856 \\
\hline & .897 & .860 & .866 & .884 & .917 & .857 & .861 & .915 \\
\hline & .947 & .947 & .955 & .943 & .960 & .957 & .955 & .94 \\
\hline hor & .786 & .899 & .907 & .861 & .911 & .927 & .910 & .890 \\
\hline & .65 & .651 & .654 & .64 & .6 & .656 & .663 & .64 \\
\hline & .758 & .842 & .837 & .810 & .797 & .837 & .842 & .817 \\
\hline o- & .736 & .838 & .845 & .859 & .726 & .828 & .832 & .859 \\
\hline & .574 & .545 & .559 & .607 & .498 & .604 & .598 & .598 \\
\hline & & .980 & .979 & .976 & .709 & .951 & .945 & .984 \\
\hline $\mathrm{v}$ & .723 & .730 & .729 & .729 & .654 & .723 & .724 & .713 \\
\hline & .966 & .958 & .959 & .963 & .937 & .948 & .950 & .950 \\
\hline & .932 & .968 & .973 & .943 & .977 & .970 & .970 & .980 \\
\hline & .926 & .960 & .965 & .939 & .943 & .959 & .964 & .956 \\
\hline & .791 & .816 & .820 & .813 & .798 & .820 & .820 & .823 \\
\hline & 3.03 & 2.45 & 1.78 & 2.73 & 2.87 & 2.45 & 2.17 & 2.52 \\
\hline & 5 & 5 & 14 & 6 & 6 & 7 & 8 & 9 \\
\hline
\end{tabular}

Looking at the mean ranks, the ordering is quite clear for J48 models; A25 is better than A10, which is better than using internal cross-validation. All three algorithms are, however, clearly better than the standard approach. From a Friedman test, the only statistically significant difference is that A25 obtained higher accuracy than the standard approach. For RBF models, the picture is again quite different. Here, the internal cross-validation is the most successful approach, even if the overall differences are very small.

The AUC results for Experiment 2 are presented in Table VII below. Again, the results are very different for J48 and the RBF networks. When using J48 models, both A10 and A25 obtained significantly higher AUC, compared to the global model. For RBF models, however, the global model has the best ranking ability followed by using internal crossvalidation, while A25 and A10 are clearly worse.

TABLE VII

EXP. 2 - AUC

\begin{tabular}{|c|c|c|c|c|c|c|c|c|}
\hline \multirow[b]{2}{*}{ Data set } & \multicolumn{4}{|c|}{$\mathrm{J} 48$} & \multicolumn{4}{|c|}{$\mathrm{RBF}$} \\
\hline & $100 \%$ & A10 & A25 & CV & $100 \%$ & A10 & A25 & $\mathrm{CV}$ \\
\hline bal. scale & .845 & .888 & .890 & .966 & .971 & .962 & .967 & .971 \\
\hline breast cancer & .606 & .632 & .626 & .632 & .687 & .634 & .621 & .641 \\
\hline oreast cancer-w & .957 & .976 & .980 & .977 & .990 & .974 & .975 & .978 \\
\hline ar & .981 & .986 & .987 & .982 & .979 & .994 & .995 & .978 \\
\hline $\mathrm{cmc}$ & .691 & .652 & .651 & .691 & .681 & .625 & .625 & .728 \\
\hline orse-colic & .843 & .829 & .829 & .844 & .835 & .821 & .825 & .809 \\
\hline edi & .886 & .887 & .889 & .882 & .875 & .877 & .878 & .868 \\
\hline & .647 & .679 & .678 & .664 & .756 & .655 & .657 & .754 \\
\hline der-bands & .500 & .752 & .750 & .733 & .808 & .711 & .710 & .802 \\
\hline 1 & .982 & .997 & .999 & .998 & .999 & .996 & .997 & .997 \\
\hline & .751 & .740 & .736 & .737 & .789 & .744 & .746 & .804 \\
\hline coli & .963 & .965 & .967 & .969 & .976 & .964 & .967 & .968 \\
\hline slass & .794 & .839 & .859 & .821 & .827 & .815 & .849 & .841 \\
\hline Ian & .568 & .626 & .640 & 610 & .652 & .568 & .585 & .614 \\
\hline ea & .769 & .846 & .852 & .820 & .900 & .850 & .853 & .891 \\
\hline ea & .775 & .827 & .821 & .823 & .887 & .789 & .831 & .871 \\
\hline a & .786 & .843 & .842 & .793 & .895 & .832 & .826 & .895 \\
\hline & .668 & .761 & .751 & .712 & .878 & .748 & .757 & .785 \\
\hline here & .891 & .871 & .870 & .885 & .954 & .867 & .850 & .954 \\
\hline & .990 & 1.000 & 1.000 & .999 & 1.000 & 1.000 & 1.000 & 1.000 \\
\hline bor & .726 & .935 & .938 & .866 & .951 & .973 & .940 & .925 \\
\hline & .650 & .647 & .652 & .633 & .680 & .646 & 648 & .674 \\
\hline hography & .785 & .870 & .864 & .877 & .868 & .854 & .857 & .814 \\
\hline sonar & .753 & .882 & .882 & .856 & .812 & .850 & .856 & .864 \\
\hline tae & .747 & .756 & .761 & .737 & .686 & .767 & .772 & .724 \\
\hline & .901 & .997 & .997 & .996 & .739 & .997 & .998 & .994 \\
\hline vehicle & .762 & .734 & .735 & .765 & .789 & .663 & .663 & .802 \\
\hline vote & .979 & .969 & .972 & .983 & .978 & .957 & .966 & .966 \\
\hline wine & .968 & .991 & .992 & .969 & .993 & .998 & .994 & .985 \\
\hline zoo & 1.000 & 1.000 & 1.000 & 1.000 & .995 & 1.000 & 1.000 & 1.000 \\
\hline & .805 & .846 & .847 & .841 & .861 & .838 & .840 & .863 \\
\hline & 3.33 & 2.20 & 2.02 & 2.45 & 1.90 & 3.15 & 2.62 & 2.33 \\
\hline \#wins & 4 & 10 & 15 & 9 & 17 & 3 & 6 & 8 \\
\hline
\end{tabular}

Summarizing Experiment 2 by comparing pair-wise wins in Table VIII below, the most important observation is probably that A10 and A25 obtained significantly higher accuracy than the global model, using either J48 trees or RBF networks. The internal cross-validation produced significantly more accurate RBF models, but was less successful for J48 models. Looking at AUCs, all three algorithms clearly outperformed the global model when using J48 models, but were actually significantly 
worse than the global model when using RBF models.

TABLE VIII

EXP. 2 - SUMMARY

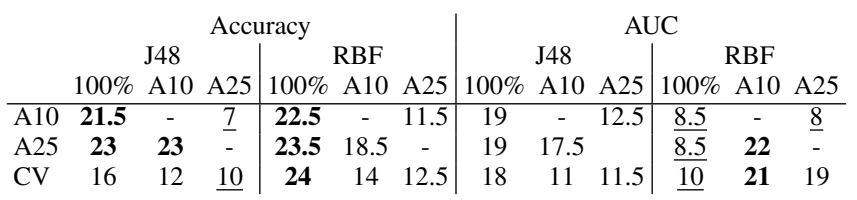

\section{CONCLUSION}

In this study, different schemes for utilizing locality when inducing predictive models, in the form of standard decision trees and RBF neural networks, have been proposed and evaluated. The main idea consists of building models specific to each test instance, using only a proportion of the available training instances, selected based on locality to the test instance at hand. In Experiment 1, different proportions (ranging from $1 \%$ to $75 \%$ ) of the training data were used to build the model for each test instance. The overall result from this experiment is that schemes using only a proportion of training data for model building generally yields better predictive performance than using all available training data and building a global model. There are, however, big variations between data sets and no setup consistently performs better than all the other. There is also a marked difference between the two techniques used, with the difference being more pronounced for J48 decision trees, than for RBF neural networks. Specifically, for decision trees, most local techniques producing J48 trees obtained significantly higher accuracy and AUC, compared to the global J48 model. For RBF networks, only local models using $50 \%$ of the training data obtained significantly higher accuracy than the global RBF model, and when measuring predictive performance as AUC, the global model actually outperformed all local models. This difference is especially interesting since $\mathrm{J} 48$ decision trees do not employ any information about locality when inducing models, whereas RBF networks utilize $k$-means clustering as a preprocessing step for its logistic regression. In Experiment 2, two different ways of finding a suitable proportion of instances to use for model building are evaluated. Using internal cross-validation on each fold to find a suitable proportion produced significantly more accurate RBF models, but was less successful for J48 trees. When using an iterative, but greedy, search procedure to determine the best proportion, models significantly more accurate than the global model were obtained both for J48 trees and RBF networks. The AUC results were again different for the two techniques, with $\mathrm{J} 48$ models found by the greedy local search being clearly better than the global model, while the global RBF model significantly outperformed all models found from the greedy search. To conclude, the experiments show that the proposed method of inducing local models improves predictive performance for both decision trees, and to a slightly lesser degree, RBF networks over a large number of data sets.
It should also be noted that the proposed method could be utilized with any type of predictive model.

\section{DISCUSSION}

Building one model for each test instance is of course quite computationally expensive. At the same time, it must be noted that this process is very parallelizable. From the results it is obvious that predictive performance can be improved by using locally trained models for each instance, but it remains to investigate for which inductive techniques this applies. Finally, the fact that very different training sizes were successful for different data sets, more sophisticated schemes to find an optimal number of neighboring training instances should be developed and evaluated.

\section{REFERENCES}

[1] C. M. Bishop, Neural Networks for Pattern Recognition. Oxford University Press, November 1995.

[2] E. A. Helgee, L. Carlsson, S. Boyer, and U. Norinder, "Evaluation of quantitative structure-activity relationship modeling strategies: local and global models." Journal of chemical information and modeling, vol. 50, no. 4, pp. 677-89, Apr. 2010.

[3] S. Zhang, A. Golbraikh, S. Oloff, H. Kohn, and A. Tropsha, "A Novel Automated Lazy Learning QSAR (ALL-QSAR) Approach: Method Development, Applications, and Virtual Screening of Chemical Databases Using Validated ALL-QSAR Models," Journal of chemical information and modeling, vol. 46, pp. 1984-1995, 2006.

[4] X. Fern and C. Brodley, "Boosting lazy decision trees," in Proceedings of the Twentieth International Conference on Machine Learning, vol. 20, no. 1,2003 , pp. 178-185.

[5] D. Margineantu and T. Dietterich, "Improved class probability estimates from decision tree models," Lecture Notes in Statistics - Nonlinear Estiamtion and Classification, vol. 171, pp. 173-188, 2003.

[6] X. Zhu, "Bagging very weak learners with lazy local learning," 2008 19th International Conference on Pattern Recognition, pp. 1-4, 2008.

[7] Y. Yan and H. Liang, "Lazy learner on decision tree for ranking," International Journal on Artificial Intelligence Tools, vol. 17, no. 1, pp. 139-158, 2008

[8] Y. C. Martin, J. L. Kofron, and L. M. Traphagen, "Do structurally similar molecules have similar biological activity?" Journal of medicinal chemistry, vol. 45, no. 19, pp. 4350-4358, 2002.

[9] J. H. Friedman, R. Kohavi, and Y. Yun, "Lazy decision trees," in National Conference on Artificial Intelligence, 1996, pp. 717-724.

[10] R. Kohavi and C. Kunz, "Option decision trees with majority votes," in In Proc. 14th International Conference on Machine Learning, 1997, pp. $161-169$.

[11] I. H. Witten and E. Frank, Data Mining: Practical Machine Learning Tools and Techniques, Second Edition (Morgan Kaufmann Series in Data Management Systems). Morgan Kaufmann, 2005.

[12] J. R. Quinlan, C4.5: programs for machine learning. Morgan Kaufmann Publishers Inc., 1993.

[13] T. Fawcett, "An introduction to roc analysis," Pattern Recognition Letters, vol. 27, no. 8, pp. 861-874, 2006.

[14] A. P. Bradley, "The use of the area under the roc curve in the evaluation of machine learning algorithms," Pattern Recognition, vol. 30, no. 7, pp. 1145-1159, 1997.

[15] A. Asuncion and D. J. Newman, "UCI machine learning repository," 2007.

[16] J. Demšar, "Statistical comparisons of classifiers over multiple data sets," J. Mach. Learn. Res., vol. 7, pp. 1-30, 2006.

[17] M. Friedman, "The use of ranks to avoid the assumption of normality implicit in the analysis of variance," Journal of American Statistical Association, vol. 32, pp. 675-701, 1937.

[18] O. J. Dunn, "Multiple Comparisons Among Means," Journal of the American Statistical Association, vol. 56, no. 293, pp. 52-64, 1961.

[19] P. B. Nemenyi, Distribution-free multiple comparisons. PhD-thesis. Princeton University, 1963. 